\title{
Synthesis and Evaluation of Some Coumarin Based Schiff's Bases as Potential Antimicrobial Agents'
}

\author{
VISHAKHA BANSODE AND MEENAKSHI N. DEODHAR*
}

Department of Pharmaceutical Chemistry, PDEA's Seth Govind Raghunath Sable College of Pharmacy, Saswad, Pune - 412301, India

mndeodhar@gmail.com

Received 30 August 2011; Accepted 28 October 2011

\begin{abstract}
A series of the title compounds 3-(4-(4, 5-dihydro-5-(substituted phenyl)-1H-3-pyrazolyl) phenylimino) methyl)-4-chloro-2 $H$-chromen-2-one $\mathbf{5}(\mathbf{a}-\mathbf{g})$ have been synthesized. These compounds were characterized on the basis of their spectral (IR, ${ }^{1} \mathrm{H}$ NMR) data and evaluated for antimicrobial activity in vitro against gram positive bacteria, gram negative bacteria and fungi. The compound (5b) was found to be the most active with MIC of 20 $\mu \mathrm{g} / \mathrm{ml}$ against all the tested organisms.
\end{abstract}

Keywords: Coumarin, Pyrazoline, Schiff's base, Antimicrobial activity.

\section{Introduction}

Development of resistance and adverse reactions towards existing antimicrobial therapy and a sharp rise in the population of immunocompromised patients has provided a thrust to the research in the area of antimicrobials. There are a number of reports that natural and synthetic coumarin derivatives possess antimicrobial activity. Novobiocin and chlorobiocin are established antimicrobials containing a coumarin skeleton. The biological effects of coumarins include antibacterial ${ }^{1}$, anti-inflammatory ${ }^{2}$, anti-AIDS ${ }^{3}$, antithrombotic ${ }^{4}$ and antitumourogenic ${ }^{4}$ effects. Similarly pyrazolines have been independently reported to possess antibacterial, antifungal ${ }^{5}$, anticancer ${ }^{6}$, antimalarial ${ }^{7}$ and anticonvulsant ${ }^{8}$ activity. In continuation of the work done in our lab on coumarin based antimicrobial agents ${ }^{9}$, we have designed and synthesized the series of compounds incorporating both, coumarin and pyrazoline moieties as potential antimicrobial agents against gram positive bacteria, gram negative bacteria and fungi.

\section{Experimental}

All the chemicals used in the synthesis were of laboratory grade. Melting points were determined in open capillary on Veego melting point electronic apparatus (VMP-D) and are uncorrected. The IR spectra of the synthesized compounds were recorded on Shimadzu 
8400-S-FTIR spectrophotometer using potassium bromide. The ${ }^{1} \mathrm{H}$ NMR spectra were recorded in $\mathrm{CDCl}_{3}$ using NMR Varian-Mercury $300 \mathrm{MHz}$ spectrometer and chemical shifts are given in units as parts per million, downfield from tetramethylsilane (TMS) as an internal standard. To monitor the reactions as well as to establish the identity and purity of reactants and products, thin layer chromatography was performed on precoated aluminium sheets (silica gel $60 \mathrm{~F}_{254}, 6 \times 2.5 \mathrm{~cm}$ ) using toluene-methanol and chloroform-ethyl acetate as appropriate solvent systems. The spots were visualized under ultra-violet light or by exposure to iodine vapors.

The target compounds were synthesized as per the following scheme:

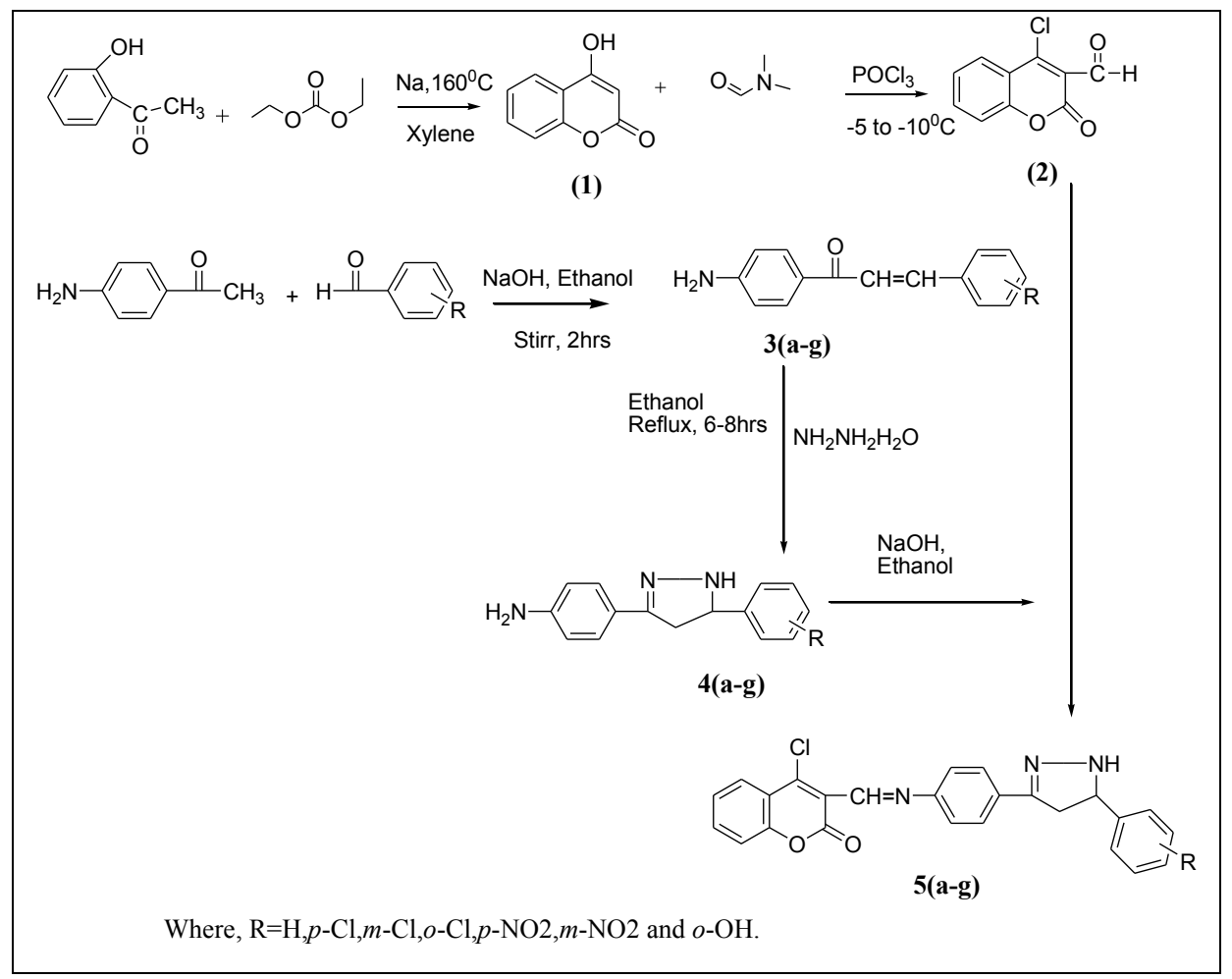

Scheme 1

General procedure for synthesis of 4-(4, 5-dihydro-5-(substituted phenyl)-1H-pyrazol-3-yl) benzenamine 4(a-g)

A mixture of 1-(4-aminophenyl)-3-(substituted phenyl)-prop-2-en-1-one 3(a-g) (0.01 mol) and hydrazine hydrate $(0.03 \mathrm{~mol})$ in $20 \mathrm{ml}$ absolute ethanol was taken in a $100 \mathrm{ml}$ RBF. The reaction mixture was heated under reflux for 6-8 hrs then left overnight at $0^{0} \mathrm{C}$. The crude 
product which separated out was filtered, washed with water and recrystalized from ethanol. The physical and the spectral data are presented in Table1.

Table 1. Physical and spectral data of 4-(4, 5-dihydro-5-(substituted phenyl)-1H-pyrazol-3-yl) benzenamine $\mathbf{4}(\mathbf{a}-\mathrm{g})$.<smiles>[R]c1ccc([C@]2([2H])NN=C(c3ccc(N)cc3)[C@H]2[2H])cc1</smiles>

\begin{tabular}{|c|c|c|c|c|c|}
\hline $\begin{array}{l}\text { Code } \\
\text { No. }\end{array}$ & $\mathbf{R}$ & $\begin{array}{c}\text { M. P.* } \\
\left({ }^{0} \mathrm{C}\right)\end{array}$ & $\begin{array}{c}\mathbf{R}_{\mathbf{f}}{ }^{*} \\
\end{array}$ & $\begin{array}{l}\text { Percentage } \\
\text { Yield } \\
(\%)\end{array}$ & $\begin{array}{c}\mathrm{IR} \\
\left(\mathrm{KBr}, \mathrm{cm}^{-1}\right)\end{array}$ \\
\hline $4 a$ & H- & $122-126$ & 0.67 & 69 & $\begin{array}{l}3446(\mathrm{NH}), 3330 \& 3319\left(\mathrm{NH}_{2}\right), 3087 \\
(\mathrm{Ar}-\mathrm{CH}), 1523 \text { (pyrazoline ring } \mathrm{C}=\mathrm{N}), \\
1500(\mathrm{C}=\mathrm{C}) .\end{array}$ \\
\hline $4 b$ & $p$-Cl- & $135-139$ & 0.71 & 60 & 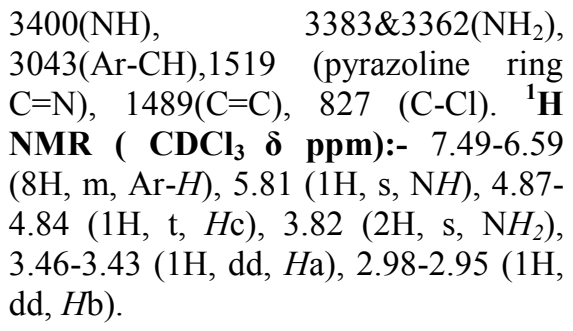 \\
\hline $4 c$ & $m-\mathrm{Cl}-$ & $137-140$ & 0.68 & 65 & $\begin{array}{l}3410(\mathrm{NH}), \quad 3334 \& \quad \& \quad 3324 \quad\left(\mathrm{NH}_{2}\right), \\
3035(\mathrm{Ar}-\mathrm{CH}), 1558 \text { (pyrazoline ring } \\
\mathrm{C}=\mathrm{N}), 1489(\mathrm{C}=\mathrm{C}), 827(\mathrm{C}-\mathrm{Cl}) .\end{array}$ \\
\hline $4 d$ & $o-\mathrm{Cl}-$ & $133-136$ & 0.66 & 68 & $\begin{array}{l}3405(\mathrm{NH}), \quad 3390 \& 3383\left(\mathrm{NH}_{2}\right), \\
2950(\mathrm{Ar}-\mathrm{CH}), 1510 \quad(\text { pyrazoline ring } \\
\mathrm{C}=\mathrm{N}), 1480(\mathrm{C}=\mathrm{C}), 829(\mathrm{C}-\mathrm{Cl}) .\end{array}$ \\
\hline $4 \mathrm{e}$ & $p-\mathrm{NO}_{2}-$ & $148-151$ & 0.62 & 78 & $\begin{array}{l}3409(\mathrm{NH}), 3345 \& 3333\left(\mathrm{NH}_{2}\right), 3036 \\
(\mathrm{Ar}-\mathrm{CH}), 1600(\text { pyrazoline ring } \mathrm{C}=\mathrm{N}), \\
1500\left(\mathrm{NO}_{2}\right), 1450(\mathrm{C}=\mathrm{C}) .\end{array}$ \\
\hline $4 \mathrm{f}$ & $m-\mathrm{NO}_{2-}$ & $145-150$ & 0.71 & 80 & $\begin{array}{l}3401(\mathrm{NH}), 3390 \& 3383\left(\mathrm{NH}_{2}\right), 3043(\mathrm{Ar}- \\
\mathrm{CH}), 1519\left(\mathrm{NO}_{2}\right), 1528(\text { pyrazoline ring } \\
\mathrm{C}=\mathrm{N}), 1450(\mathrm{C}=\mathrm{C}) .\end{array}$ \\
\hline $4 g$ & $o-\mathrm{OH}-$ & $125-128$ & 0.65 & 74 & $\begin{array}{l}3401(\mathrm{NH}), 3360 \& 3334\left(\mathrm{NH}_{2}\right), 3200(\mathrm{Ar}- \\
\mathrm{OH}), 3035(\mathrm{Ar}-\mathrm{CH}), 1604(\text { pyrazoline } \\
\text { ringC }=\mathrm{N}), 1496(\mathrm{C}=\mathrm{C})\end{array}$ \\
\hline
\end{tabular}

*All melting points are uncorrected, **Mobile phase- Benzene: Ethyl acetate $=9: 1$, Recrystalization solvent: Ethanol. 
General procedure for synthesis of 3-(4-(4, 5-dihydro-5-(substituted phenyl)-1H-pyrazolyl) phenylimino) methyl)-4-chloro-2H-chromen-2-one (5a-g)

A mixture of 4-(4, 5-dihydro-5-(substituted phenyl)-1H-pyrazol-3-yl) benzenamine 4(a-g) $(0.01 \mathrm{~mol})$ and 4-chloro-2-oxo- $2 H$-chromene-3-carbaldehyde (2) $(0.01 \mathrm{~mol})$ was taken in a $100 \mathrm{ml}$ RBF containing $20 \mathrm{ml}$ of ethanol. Ethanolic $\mathrm{NaOH}(10 \%, 10 \mathrm{ml})$ was added to the reaction mixture $\&$ it was heated under reflux for 6-8 hrs after which it was allowed to cool to room temperature. The solid obtained was filtered, washed with water, dried in air and recrystallized from ethanol. The physical and the spectral data are presented in Table 2.

\section{Antimicrobial activity}

Zone of inhibition (Cup-plate method)

All the target compounds 5(a-g) were tested for their antimicrobial activity by agar cup plate method $^{10}$. The organisms used for antibacterial activity were gram positive bacteria: $S$. aureus (ATCC 9144), B. subtilis (ATCC 6633) and S. epidermis (ATCC 12228), gram negative bacteria: E. coli (ATCC 25922), S. typhi and P. aeruginosa (ATCC 9027) and the media was nutrient agar broth. The antifungal activity was performed against $A$. niger (ATCC 10594) and C. albicans (ATCC 10231). The media being potato dextrose agar. Amoxycillin and fluconazole were used as standards for antibacterial and antifungal activity respectively. The observations are given in Table $\mathbf{3 , 4}$ and $\mathbf{5}$ for gram positive bacteria, gram negative bacteria and fungi respectively.

Table 2. Physical and spectral data of 3-(4-(4, 5-dihydro-5-(substituted phenyl)-1H-pyrazolyl) phenylimino) methyl)-4-chloro- $2 H$-chromen-2-one (5a-g).

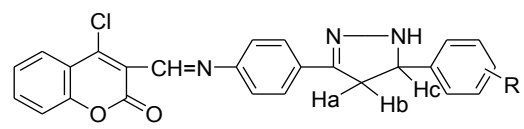

\begin{tabular}{|c|c|c|c|c|c|c|}
\hline $\begin{array}{c}\text { Code } \\
\text { No. }\end{array}$ & $\mathbf{R}$ & $\begin{array}{l}\text { M.P. }{ }^{*} \\
\left({ }^{0} \mathrm{C}\right)\end{array}$ & $\begin{array}{l}* * \\
\mathbf{R}_{\mathbf{f}}\end{array}$ & $\begin{array}{l}\text { Percentage } \\
\text { yield } \\
(\%)\end{array}$ & $\begin{array}{c}\text { I.R. } \\
\left(\mathrm{KBr}, \mathrm{cm}^{-1}\right)\end{array}$ & $\begin{array}{c}{ }^{1} \mathrm{H} \text { NMR } \\
\left(\mathrm{CDCl}_{3}, \delta p p m\right)\end{array}$ \\
\hline $5 a$ & H- & $208-210$ & 0.68 & 71 & $\begin{array}{l}3397(\mathrm{NH}), \quad 2922(\mathrm{Ar}- \\
\mathrm{CH}), \quad 1660(\text { coumarin } \\
\mathrm{C}=\mathrm{O}), \quad 1630(\mathrm{CH}=\mathrm{N}), \\
1602(\text { pyrazoline ring } \\
\mathrm{C}=\mathrm{N}), \quad 1463(\mathrm{C}=\mathrm{C}), \\
1190(\text { coumarin } \mathrm{C}-\mathrm{O}), \\
780(\mathrm{C}-\mathrm{Cl}) .\end{array}$ & $\begin{array}{l}\text { 9.21(1H,s,CH=N),8.48- } \\
8.02(12 \mathrm{H}, \mathrm{m}, \mathrm{Ar}-H), \\
6.18-6.16(1 \mathrm{H}, \mathrm{s}, \mathrm{N} H), \\
4.98-4.91(1 \mathrm{H}, \mathrm{t}, H \mathrm{c}), \\
3.66-3.69(1 \mathrm{H}, \mathrm{dd}, H \mathrm{a}), \\
3.15-3.13(1 \mathrm{H}, \mathrm{dd}, H \mathrm{~b}) .\end{array}$ \\
\hline
\end{tabular}




\begin{tabular}{|c|c|c|c|c|c|}
\hline$p-\mathrm{Cl}-$ & $225-227$ & 0.69 & 60 & $\begin{array}{l}3403(\mathrm{NH}), 3030 \quad(\text { Ar- } \\
\mathrm{CH}), 1650(\mathrm{C}=\mathrm{O}), \\
1603(\mathrm{CH}=\mathrm{N}), \\
(\text { pyrazoline ring } \mathrm{C}=\mathrm{N}), \\
1461(\mathrm{C}=\mathrm{C}), \\
1204(\text { coumarin } \quad \mathrm{C}-\mathrm{O}), \\
780(\mathrm{C}-\mathrm{Cl})\end{array}$ & $\begin{array}{l}9.12(1 \mathrm{H}, \mathrm{s}, \mathrm{C} H=\mathrm{N}), 8.79- \\
7.24(12 \mathrm{H}, \mathrm{m}, \mathrm{Ar}-H), \\
6.22(1 \mathrm{H}, \mathrm{s}, \mathrm{N} H), 5.41- \\
5.39(1 \mathrm{H}, \mathrm{t}, H \mathrm{c}), 3.94- \\
3.91(1 \mathrm{H}, \mathrm{dd}, H \mathrm{a}), \\
3.22-3.19(1 \mathrm{H}, \mathrm{dd}, H \mathrm{~b})\end{array}$ \\
\hline$m-\mathrm{Cl}-$ & $228-230$ & 0.72 & 62 & $\begin{array}{l}3401(\mathrm{NH}), \quad 3064(\text { Ar- } \\
\mathrm{CH}), \quad 1662(\text { coumarin } \\
\mathrm{C}=\mathrm{O}), 1605 \quad(\text { pyrazoline } \\
\text { ring } \quad \mathrm{C}=\mathrm{N}), \\
1583(\mathrm{CH}=\mathrm{N}), \\
1461(\mathrm{C}=\mathrm{C}), 1217 \\
(\text { coumarin } \quad \mathrm{C}-\mathrm{O}), \quad 777 \\
(\mathrm{C}-\mathrm{Cl})\end{array}$ & $\begin{array}{l}9.12(1 \mathrm{H}, \mathrm{s}, \mathrm{C} H=\mathrm{N}), 8.79- \\
7.24(12 \mathrm{H}, \mathrm{m}, \mathrm{Ar}-H) \\
6.22(1 \mathrm{H}, \mathrm{s}, \mathrm{N} H), 5.41- \\
5.39(1 \mathrm{H}, \mathrm{t}, H \mathrm{c}), 3.94- \\
3.91(1 \mathrm{H}, \mathrm{dd}, H \mathrm{a}), \\
3.22-3.19(1 \mathrm{H}, \mathrm{dd}, H \mathrm{~b})\end{array}$ \\
\hline$o-\mathrm{Cl}-$ & $221-224$ & 0.66 & 64 & $\begin{array}{l}3401(\mathrm{NH}), 3064 \text { (Ar- } \\
\mathrm{CH}), 1662(\text { coumarin } \\
\mathrm{C}=\mathrm{O}), 1605(\text { pyrazoline } \\
\text { ring } \\
1583(\mathrm{CH}=\mathrm{N}), \\
1461(\mathrm{C}=\mathrm{C}), \\
(\text { coumarin }), \quad \mathrm{C}-\mathrm{O}), \quad 777 \\
(\mathrm{C}-\mathrm{Cl})\end{array}$ & $\begin{array}{l}9.12(1 \mathrm{H}, \mathrm{s}, \mathrm{C} H=\mathrm{N}), 8.79- \\
7.24(12 \mathrm{H}, \mathrm{m}, \mathrm{Ar}-H), \\
6.22(1 \mathrm{H}, \mathrm{s}, \mathrm{N} H), 5.41- \\
5.39(1 \mathrm{H}, \mathrm{t}, H \mathrm{c}), 3.94- \\
3.91(1 \mathrm{H}, \mathrm{dd}, H \mathrm{a}), \\
3.22-3.19(1 \mathrm{H}, \mathrm{dd}, H \mathrm{~b})\end{array}$ \\
\hline$p-\mathrm{NO}_{2-}$ & $230-233$ & 0.65 & 74 & $\begin{array}{l}3390(\mathrm{NH}), 2925(\text { Ar- } \\
\mathrm{CH}), \quad 1634(\text { coumarin } \\
\mathrm{C}=\mathrm{O}), \quad 1583(\mathrm{CH}=\mathrm{N}), \\
1558(\text { pyrazoline ring }) \\
1500\left(\mathrm{NO}_{2}\right), 1461(\mathrm{C}=\mathrm{C}), \\
1241(\text { coumarin } \quad \mathrm{C}-\mathrm{O}), \\
780(\mathrm{C}-\mathrm{Cl}),\end{array}$ & $\begin{array}{l}9.291 \mathrm{H}, \mathrm{s}, \mathrm{C} H=\mathrm{N}), 8.87- \\
7.21(12 \mathrm{H}, \mathrm{m}, \mathrm{Ar}-H), \\
6.23(1 \mathrm{H}, \mathrm{s}, \mathrm{N} H), 5.34- \\
5.32(1 \mathrm{H}, \mathrm{t}, H \mathrm{c}), 3.41- \\
3.39(1 \mathrm{H}, \mathrm{dd}, H \mathrm{a}), \\
2.52-5.54(1 \mathrm{H}, \mathrm{dd}, H \mathrm{~b}) .\end{array}$ \\
\hline$m-\mathrm{NO}_{2}-$ & $229-232$ & 0.67 & 76 & $\begin{array}{l}3402(\mathrm{NH}), \quad 3076(\text { Ar- } \\
\mathrm{CH}), \quad 1644(\text { coumarin } \\
\mathrm{C}=\mathrm{O}), \\
1658(\mathrm{CH}=\mathrm{N}), 1555 \\
(\text { pyrazoline ring } \mathrm{C}=\mathrm{N}), \\
1489(\mathrm{C}=\mathrm{C}), \\
1265(\text { coumarin } \quad \mathrm{C}-\mathrm{O}), \\
1510\left(\mathrm{NO}_{2}\right), 768(\mathrm{C}-\mathrm{Cl})\end{array}$ & $\begin{array}{l}9.291 \mathrm{H}, \mathrm{s}, \mathrm{C} H=\mathrm{N}), 8.87- \\
7.21(12 \mathrm{H}, \mathrm{m}, \mathrm{Ar}-H) \\
6.23(1 \mathrm{H}, \mathrm{s}, \mathrm{N} H), 5.34- \\
5.32(1 \mathrm{H}, \mathrm{t}, H \mathrm{c}), 3.41- \\
3.39(1 \mathrm{H}, \mathrm{dd}, H \mathrm{a}), \\
2.52-5.54(1 \mathrm{H}, \mathrm{dd}, H \mathrm{~b})\end{array}$ \\
\hline$o-\mathrm{OH}-$ & $229-241$ & 0.70 & 68 & $\begin{array}{l}3460(\mathrm{Ar}-\mathrm{OH}), \\
3319(\mathrm{NH}), \quad 2876(\mathrm{Ar}- \\
\mathrm{CH}), \quad 1624(\text { coumarin } \\
\mathrm{C}=\mathrm{O}), 1560(\text { pyrazoline } \\
\text { ring } \\
\mathrm{CH}=\mathrm{N}), 1532(\mathrm{C}=\mathrm{N}), \\
1490(\mathrm{C}=\mathrm{C}), \\
1220(\text { coumarin } \mathrm{C}-\mathrm{O}), \\
790(\mathrm{C}-\mathrm{Cl})\end{array}$ & $\begin{array}{l}9.20(1 \mathrm{H}, \mathrm{s}, \mathrm{C} H=\mathrm{N}), 8.83- \\
7.21(12 \mathrm{H}, \mathrm{m}, \mathrm{Ar}-H), \\
6.69(1 \mathrm{H}, \mathrm{s}, \mathrm{Ar}-\mathrm{O} H) \\
, 6.23(1 \mathrm{H}, \mathrm{s}, \mathrm{N} H), 5.34- \\
5.32(1 \mathrm{H}, \mathrm{t}, H \mathrm{c}), 3.41- \\
3.39(1 \mathrm{H}, \mathrm{dd}, H \mathrm{a}), \\
2.52-2.54(1 \mathrm{H}, \mathrm{dd}, H \mathrm{~b}) .\end{array}$ \\
\hline
\end{tabular}

*All melting points are uncorrected, **Mobile phase- Benzene: ethyl acetate $=9: 1$, Recrystalization solvent: Ethanol. 
Table 3. Zone of inhibition against Gram positive bacteria:

\begin{tabular}{|c|c|c|c|c|}
\hline \multirow{2}{*}{$\begin{array}{l}\text { Compound } \\
\text { code }\end{array}$} & \multirow{2}{*}{$\begin{array}{c}\text { Concentration } \\
\mu \mathrm{g} / 10 \mu \mathrm{l}\end{array}$} & \multicolumn{3}{|c|}{ Zone of inhibition in mm (SEM) } \\
\hline & & S. aureus & $\begin{array}{c}\text { S. epidermis } \\
\text { epidermisepider } \\
\text { mis }\end{array}$ & B. subtilis \\
\hline Amoxycillin & 30 & $26( \pm 1.46)$ & $27( \pm 1.39)$ & $29( \pm 1.42)$ \\
\hline \multirow[t]{3}{*}{$5 \mathrm{a}$} & 50 & $6( \pm 1.37)$ & $7( \pm 1.42)$ & $5( \pm 1.44)$ \\
\hline & 100 & $10( \pm 1.51)$ & $13( \pm 1.53)$ & $9( \pm 1.50)$ \\
\hline & 150 & $13( \pm 1.36)$ & $18( \pm 1.41)$ & $14( \pm 1.62)$ \\
\hline \multirow[t]{3}{*}{$5 b$} & 50 & $9( \pm 1.39)$ & $7( \pm 1.40)$ & $7( \pm 1.50)$ \\
\hline & 100 & $14( \pm 1.40)$ & $13( \pm 1.38)$ & $15( \pm 1.52)$ \\
\hline & 150 & $25( \pm 1.52)$ & $24( \pm 1.54)$ & $27( \pm 1.61)$ \\
\hline \multirow[t]{3}{*}{$5 \mathrm{c}$} & 50 & $7( \pm 1.42)$ & $9( \pm 1.36)$ & $9( \pm 1.41)$ \\
\hline & 100 & $13( \pm 1.44)$ & $12( \pm 1.37)$ & $11( \pm 1.45)$ \\
\hline & 150 & $21( \pm 1.63)$ & $19( \pm 1.55)$ & $18( \pm 1.47)$ \\
\hline \multirow[t]{3}{*}{$5 d$} & 50 & $8( \pm 1.49)$ & $5( \pm 1.49)$ & $6( \pm 1.37)$ \\
\hline & 100 & $12( \pm 1.38)$ & $10( \pm 1.52)$ & $10( \pm 1.39)$ \\
\hline & 150 & $17( \pm 1.52)$ & $14( \pm 1.44)$ & $16( \pm 1.64)$ \\
\hline \multirow[t]{3}{*}{$5 e$} & 50 & $6( \pm 1.38)$ & $6( \pm 1.41)$ & $8( \pm 1.47)$ \\
\hline & 100 & $11( \pm 1.48)$ & $11( \pm 1.45)$ & $12( \pm 1.49)$ \\
\hline & 150 & $16( \pm 1.38)$ & $16( \pm 1.42)$ & $17( \pm 1.43)$ \\
\hline \multirow[t]{3}{*}{$5 f$} & 50 & $7( \pm 1.50)$ & $4( \pm 1.55)$ & $7( \pm 1.44)$ \\
\hline & 100 & $15( \pm 1.54)$ & $9( \pm 1.41)$ & $11( \pm 1.37)$ \\
\hline & 150 & $20( \pm 1.60)$ & $13( \pm 1.51)$ & $14( \pm 1.66)$ \\
\hline \multirow[t]{3}{*}{$5 \mathrm{~g}$} & 50 & $6( \pm 1.38)$ & $5( \pm 1.36)$ & $6( \pm 1.57)$ \\
\hline & 100 & $12( \pm 1.40)$ & $8( \pm 1.46)$ & $11( \pm 1.59)$ \\
\hline & 150 & $16( \pm 1.65)$ & $13( \pm 1.46)$ & $16( \pm 1.54)$ \\
\hline
\end{tabular}


Table 4. Zone of inhibition against Gram negative bacteria:

\section{Compound Concentration Zone of inhibition in mm(SEM) code}

$$
\mu \mathrm{g} / 10 \mu \mathrm{l}
$$

E. coli

\begin{tabular}{|c|c|c|c|c|}
\hline & & & & \\
\hline Amoxycillin & 30 & $27( \pm 1.37)$ & $25( \pm 1.35)$ & $28( \pm 1.30)$ \\
\hline \multirow[t]{3}{*}{$5 a$} & 50 & $9( \pm 1.40)$ & $9( \pm 1.42)$ & $8( \pm 1.53)$ \\
\hline & 100 & $14( \pm 1.37)$ & $13( \pm 1.50)$ & $15( \pm 1.60)$ \\
\hline & 150 & $15( \pm 1.47)$ & $15( \pm 1.49)$ & $17( \pm 1.39)$ \\
\hline \multirow[t]{3}{*}{$5 b$} & 50 & $10( \pm 1.37)$ & $10( \pm 1.38)$ & $10( \pm 1.39)$ \\
\hline & 100 & $15( \pm 1.51)$ & $14( \pm 1.44)$ & $16( \pm 1.45)$ \\
\hline & 150 & $26( \pm 1.45)$ & $20( \pm 1.48)$ & $25( \pm 1.50)$ \\
\hline \multirow[t]{3}{*}{$5 c$} & 50 & $10( \pm 1.44)$ & $6( \pm 1.46)$ & $10( \pm 1.42)$ \\
\hline & 100 & $12( \pm 1.54)$ & $12( \pm 1.38)$ & $13( \pm 1.53)$ \\
\hline & 150 & $19( \pm 1.52)$ & $19( \pm 1.48)$ & $20( \pm 1.38)$ \\
\hline \multirow[t]{3}{*}{$5 d$} & 50 & $8( \pm 1.55)$ & $9( \pm 1.49)$ & $7( \pm 1.42)$ \\
\hline & 100 & $10( \pm 1.45)$ & $12( \pm 1.51)$ & $11( \pm 1.36)$ \\
\hline & 150 & $15( \pm 1.44)$ & $18( \pm 1.37)$ & $15( \pm 1.51)$ \\
\hline \multirow[t]{3}{*}{$5 \mathrm{e}$} & 50 & $5( \pm 1.44)$ & $7( \pm 1.54)$ & $9( \pm 1.39)$ \\
\hline & 100 & $11( \pm 1.59)$ & $10( \pm 1.40)$ & $10( \pm 1.41)$ \\
\hline & 150 & $17( \pm 1.62)$ & $13( \pm 1.66)$ & $14( \pm 1.45)$ \\
\hline \multirow[t]{3}{*}{$5 f$} & 50 & $6( \pm 1.60)$ & $9( \pm 1.39)$ & $8( \pm 1.50)$ \\
\hline & 100 & $8( \pm 1.45)$ & $12( \pm 1.44)$ & $12( \pm 1.59)$ \\
\hline & 150 & $10( \pm 1.41)$ & $15( \pm 1.45)$ & $16( \pm 1.39)$ \\
\hline \multirow[t]{3}{*}{$5 \mathrm{~g}$} & 50 & $4( \pm 1.52)$ & $4( \pm 1.57)$ & $7( \pm 1.63)$ \\
\hline & 100 & $9( \pm 1.46)$ & $7( \pm 1.61)$ & $10( \pm 1.58)$ \\
\hline & 150 & $12( \pm 1.35)$ & $9( \pm 1.47)$ & $14( \pm 1.34)$ \\
\hline
\end{tabular}


Table 5. Antifungal activity.

\begin{tabular}{|c|c|c|c|}
\hline \multirow[t]{2}{*}{ Compound code } & \multirow{2}{*}{$\begin{array}{c}\text { Concentration } \\
\mu \mathrm{g} / 10 \mu \mathrm{l}\end{array}$} & \multicolumn{2}{|c|}{ Zone of inhibition in $\mathrm{mm}$ (SEM) } \\
\hline & & A. niger & C. albicans \\
\hline Fluconazole & 30 & $27( \pm 1.39)$ & $29( \pm 1.42)$ \\
\hline \multirow[t]{3}{*}{$5 \mathrm{a}$} & 50 & $6( \pm 1.42)$ & $7( \pm 1.36)$ \\
\hline & 100 & $11( \pm 1.39)$ & $13( \pm 1.40)$ \\
\hline & 150 & $14( \pm 1.51)$ & $16( \pm 1.55)$ \\
\hline \multirow[t]{3}{*}{$5 b$} & 50 & $9( \pm 1.32)$ & $10( \pm 1.34)$ \\
\hline & 100 & $15( \pm 1.39)$ & $16( \pm 1.40)$ \\
\hline & 150 & $25( \pm 1.46)$ & $27( \pm 1.36)$ \\
\hline \multirow[t]{3}{*}{$5 \mathrm{c}$} & 50 & $8( \pm 1.44)$ & $10( \pm 1.47)$ \\
\hline & 100 & $15( \pm 1.48)$ & $17( \pm 1.43)$ \\
\hline & 150 & $21( \pm 1.47)$ & $23( \pm 1.38)$ \\
\hline \multirow[t]{3}{*}{$5 d$} & 50 & $9( \pm 1.50)$ & $11( \pm 1.52)$ \\
\hline & 100 & $13( \pm 1.42)$ & $14( \pm 1.36)$ \\
\hline & 150 & $18( \pm 1.62)$ & $19( \pm 1.51)$ \\
\hline \multirow[t]{3}{*}{$5 \mathrm{e}$} & 50 & $7( \pm 1.37)$ & $8( \pm 1.41)$ \\
\hline & 100 & $10( \pm 1.42)$ & $11( \pm 1.43)$ \\
\hline & 150 & $16( \pm 1.53)$ & $17( \pm 1.52)$ \\
\hline \multirow[t]{3}{*}{$5 f$} & 50 & $6( \pm 1.55)$ & $7( \pm 1.51)$ \\
\hline & 100 & $11( \pm 1.37)$ & $12( \pm 1.38)$ \\
\hline & 150 & $14( \pm 1.46)$ & $16( \pm 1.44)$ \\
\hline \multirow[t]{3}{*}{$5 \mathrm{~g}$} & 50 & $6( \pm 1.42)$ & $7( \pm 1.36)$ \\
\hline & 100 & $11( \pm 1.39)$ & $13( \pm 1.40)$ \\
\hline & 150 & $14( \pm 1.51)$ & $16( \pm 1.55)$ \\
\hline
\end{tabular}

Minimum inhibitory concentration

The minimum inhibitory concentration (MIC) against the above mentioned organisms was determined by the broth dilution method ${ }^{11}$. The results are given in Table 6. 
Table 6. Minimum inhibitory concentration for Gram positive \& Gram negative bacteria:-

\begin{tabular}{|c|c|c|c|c|c|c|}
\hline \multirow[t]{2}{*}{$\begin{array}{l}\text { Compound } \\
\text { Code }\end{array}$} & \multicolumn{3}{|c|}{$\begin{array}{c}\text { MIC } \\
(\mu \mathrm{g} / \mathrm{ml})\end{array}$} & \multicolumn{3}{|c|}{$\begin{array}{c}\text { MIC } \\
(\mu \mathrm{g} / \mathrm{ml})\end{array}$} \\
\hline & B. subtilis & $\begin{array}{l}\text { S. aureus } \\
\text { B.subtilis }\end{array}$ & S. epidermis & E. coli & $\begin{array}{l}\text { P. aeruginosa } \\
\text { aaaeruginosa }\end{array}$ & S. typhi \\
\hline $5 a$ & 25 & 25 & 30 & 25 & 30 & 30 \\
\hline $5 b$ & 20 & 20 & 20 & 20 & 20 & 20 \\
\hline $5 c$ & 20 & 25 & 35 & 30 & 35 & 25 \\
\hline $5 d$ & 25 & 30 & 35 & 35 & 40 & 30 \\
\hline $5 \mathrm{e}$ & 30 & 35 & 30 & 35 & 30 & 25 \\
\hline $5 f$ & 25 & 30 & 30 & 30 & 25 & 35 \\
\hline $5 \mathrm{~g}$ & 30 & 25 & 30 & 35 & 30 & 35 \\
\hline
\end{tabular}

\section{Results and Discussion}

4-Hydroxycoumarin (1) ${ }^{12}$, 4-chloro-2-oxo-2H-chromene-3-carbaldehyde (2) ${ }^{13}$ and 1-(4aminophenyl)-3-(substituted phenyl) prop-2-en-1-ones 3(a-g), ${ }^{14}$ and ${ }^{15}$ were synthesized by using reported procedures. The physical and the spectral values were found to match with the reported values.

4-(4, 5-Dihydro-1-phenyl-5-(substituted phenyl)-1H-pyrazol-3-yl) benzene-amine 4 (a-g) were synthesized by reaction of 1-(4-aminophenyl)-3-(substituted phenyl) prop-2-en-1-one 3(a-g) with hydrazine hydrate using ethanol as solvent. These were then reacted with 4chloro-2-oxo- $2 H$-chromene-3-carbaldehyde (2) to yield the target compounds 3-(4-(4, 5dihydro-5-(substituted phenyl)-1 $H$-3-pyrazolyl) phenylimino) methyl)-4-chloro- $2 \mathrm{H}$ chromen-2-one (5a-g).

All the synthesized compounds $\mathbf{4}(\mathbf{a}-\mathbf{g})$ were solids melting at around the range of $122-151^{\circ} \mathrm{C}$ (Table 1) and freely soluble in acetone and chloroform. The solid state IR (KBr) spectra of these compounds reveal a characteristic aromatic stretch between $3087-2950 \mathrm{~cm}^{-1}$. The NH group present in the pyrazoline ring revealed peaks at around the range $3400-3446 \mathrm{~cm}^{-1}$. The $\mathrm{C}=\mathrm{N}$ group present in the pyrazoline ring peaks were observed at around the range 1510$1604 \mathrm{~cm}^{-1}$. The stretching vibrations for free amino group are seen between $3319-3390 \mathrm{~cm}^{-1}$ (Table 1). The ${ }^{1} \mathrm{H}$ NMR spectrum of the compound (4b) was recorded in $\mathrm{CDCl}_{3}$. The compound (4b) revealed doublet at around 4.87-4.84 ppm for pyrazoline 5- $H$ and singlet for $\mathrm{NH}$ at $5.81 \mathrm{ppm}$. The pyrazoline 4- $\mathrm{CH}_{2}$ revealed a doublet of doublet at around 3.46-2.95 ppm. The singlet for $\mathrm{NH}_{2}$ was observed at $3.82 \mathrm{ppm}$. The aromatic protons showed peaks at 7.30-7.49 \& 6.68-6.5 ppm as multiplets (Table 1). All the target compounds 5(a-g) were solids melting at around the range of $208-241{ }^{\circ} \mathrm{C}$ and freely soluble in acetone and chloroform. The solid state IR $\left(\mathrm{KBr}, \mathrm{cm}^{-1}\right)$ spectra of these compounds reveal a characteristic aromatic stretch between 2876-3076 $\mathrm{cm}^{-1}$ and sharp carbonyl stretching vibration for coumarin lactone at $1624-1662 \mathrm{~cm}^{-1}$ (Table 2). The $-\mathrm{CH}=\mathrm{N}$ stretch of was observed at 1560$1658 \mathrm{~cm}^{-1}$. No peak was observed for free amino group observed in the spectra of 4(a-g) at around $3300-3200 \mathrm{~cm}^{-1}$ indicating incorporation of this group into the Schiff's base.

The ${ }^{1} \mathrm{H}$ NMR spectra of the series of target compounds $\mathbf{5}(\mathbf{a}-\mathbf{g})$ were recorded in $\mathrm{CDCl}_{3}$. All compounds revealed a doublet of doublet for pyrazoline $5-H$ at around $4.98-4.91 \mathrm{ppm}$. The $\mathrm{CH}=\mathrm{N}$ proton appeared at 9.29-9.12 ppm. (Table 2). The aromatic protons showed peaks at 8.87-7.29 ppm as multiplets. No peak was observed for free amino group observed in the 
spectra of $4(\mathbf{a}-\mathrm{g})$ at $3.82 \mathrm{ppm}$ confirms the formation of $-\mathrm{CH}=\mathrm{N}$ group. This indicates the successful condensation of 4-(4, 5-dihydro-phenyl-5-(substituted phenyl)-1 $H$-pyrazol-3-yl) benzenamine 4(a-g) with 4-chloro-2-oxo- $2 H$-chromene-3-carbaldehyde (2).

All the target compounds have shown good antimicrobial activity (Table no. 3, 4, 5 and 6) as compared to standard and compound $\mathbf{5 b}$ exhibiting highest zone of inhibition and least MIC $(20 \mu \mathrm{g} / 10 \mu \mathrm{l}))$ against all the organisms.

\section{Conclusion}

From the antimicrobial data of the synthesized compounds, we can conclude that the series of target compounds 5(a-g), 4-chloro-2-oxo- $2 \mathrm{H}$-chromene-3-carbaldehyde and 4- (4, 5dihydro-phenyl-5-(substituted phenyl)-1H-pyrazol-3-yl) benzenamines hold promise as potential antimicrobial agents after further optimization.

\section{Acknowledgement}

Authors are thankful to Dr. Ashok V. Bhosale, Principal, PDEA's S. G. R. S. College of Pharmacy for providing us the required facilities for the completion of project.

\section{References}

1. Rajendraprasad Y, Ravikumar P, Ashadeepti C and Venkataramanae H, E. J. Chem., 2006, 3, 236.

2. Omaima M, Kamelia M, Rasha Z, Batran T and Maher S, Bioorg. Med. Chem., 2010, 18, 3371 .

3. Jalpa C, Jitender B, Kuldip D and Upadhyay Y, Tetrahedron Lett., 2007, 48, 8472.

4. Koneni V, Abdhesh K, Manoj K, Jayanta S and Sinha S, Bioorg. Med. Chem. Lett., 2010, 20, 7205.

5. Andrea S and Abonia R, Bioorg. Med. Chem. Lett., 2010, 15, 2834.

6. Insuasty B, García A, Quiroga J, Ortiz A and Nogueras M, Eur. J. Med. Chem., 2011, 46, 2436.

7. Wanare G, Aher R, Kawathekar N, Ranjan R, Kaushik N and Sahal D, Bioorg. Med. Chem. Lett., 2010, 20, 4675.

8. Amnerkar N and Bhusari K, Eur. J. Med. Chem., 2010, 45,149.

9. Bairagi S, Deodhar M and Bhosale A, E. J. Chem., 2009, 6, 759.

10. Hugo W and Russell A, Pharmaceutical microbiology, $8^{\text {th }}$ ed., Blackwell Science Ltd.: United Kingdom, 1998, 268.

11. Kokare C, Pharmaceutical microbiology, Experiments and techniques, $2^{\text {nd }}$ ed., Career Publications, Nashik: India, 2007, 138.

12. Braccio M, Grossi G, Roma G, Signorello M and Leancini G, Eur. J. Med. Chem., 2004,39, 337.

13. Rajanna K, Florence S, and Ali M, lnt. J. Chem. Kin., 1996, 28, 865.

14. Kaufmann W, Adams R, J. Am. Chem. Soc., 1923, 45, 1839.

15. Emerson W. Patrick T, J. Org. Chem., 1949, 14, 790. 


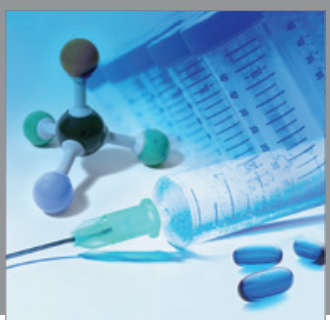

International Journal of

Medicinal Chemistry

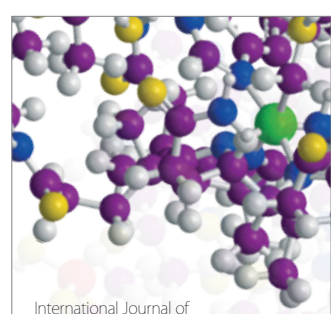

Carbohydrate Chemistry

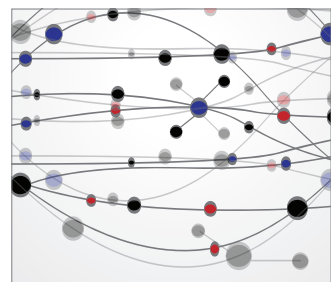

The Scientific World Journal
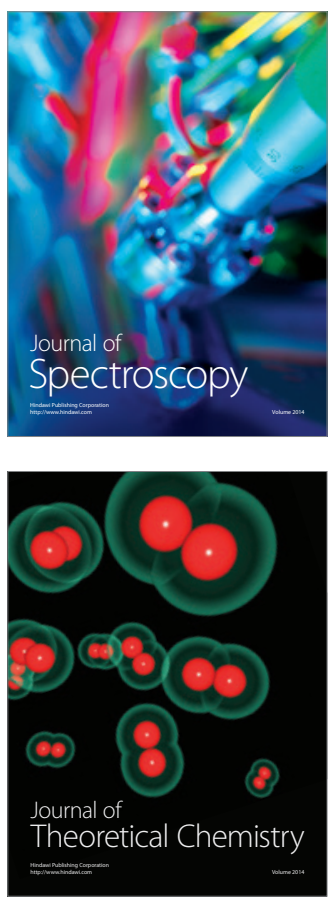
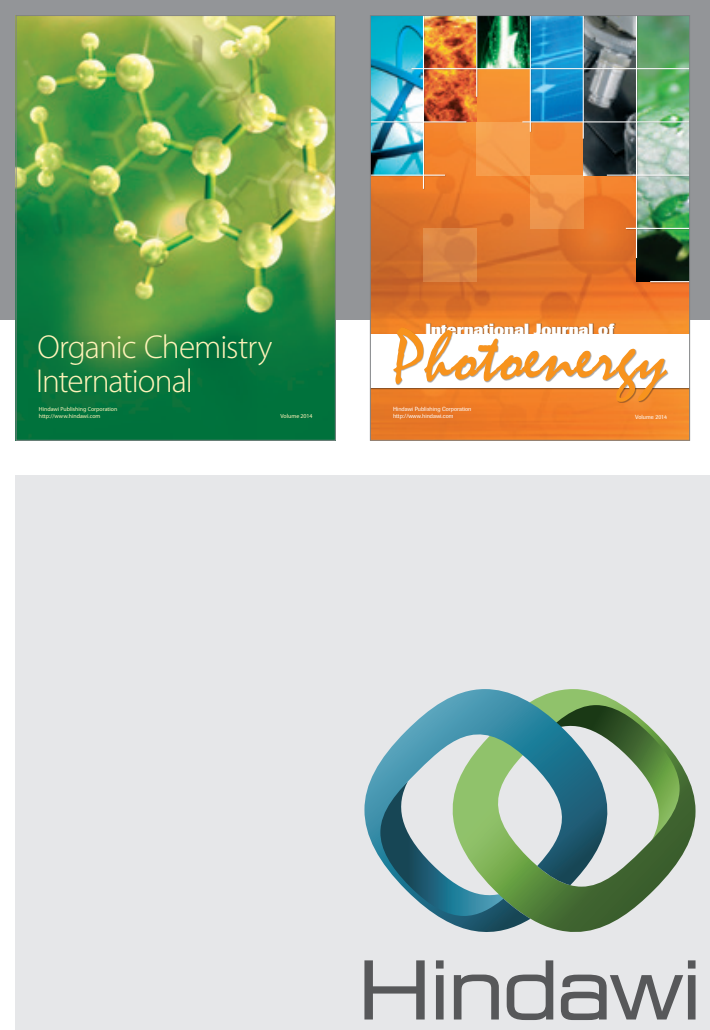

Submit your manuscripts at

http://www.hindawi.com
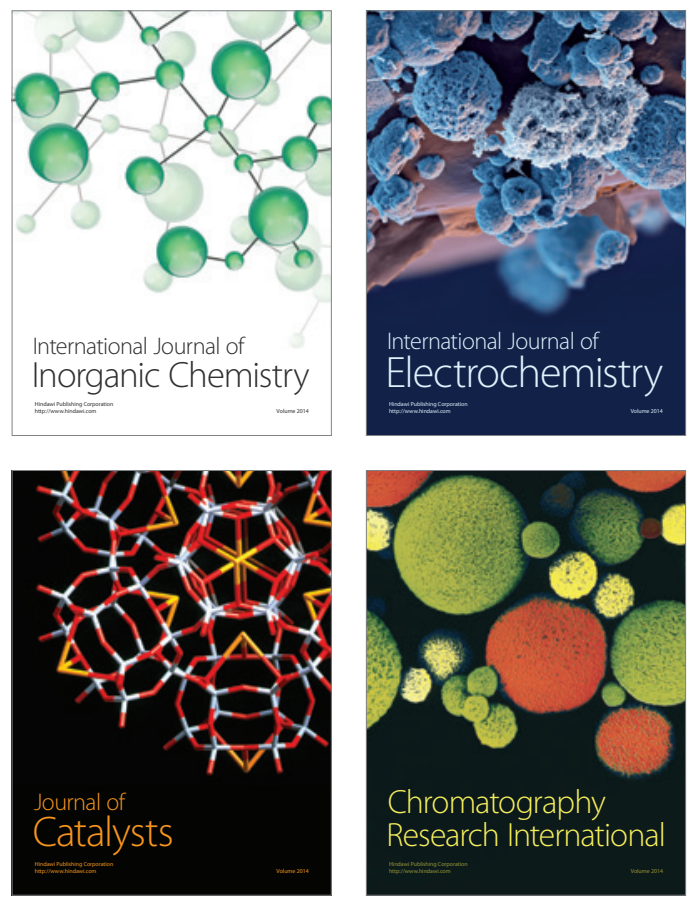
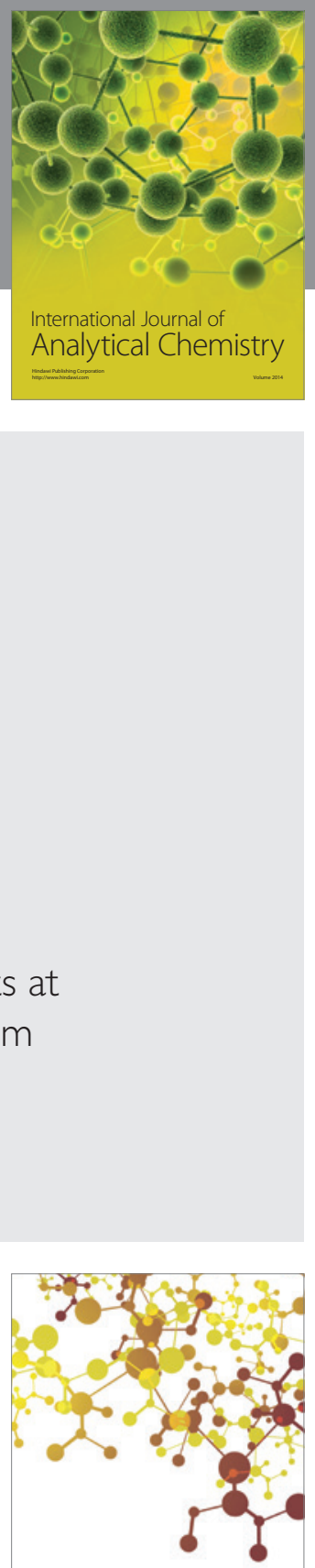

Journal of

Applied Chemistry
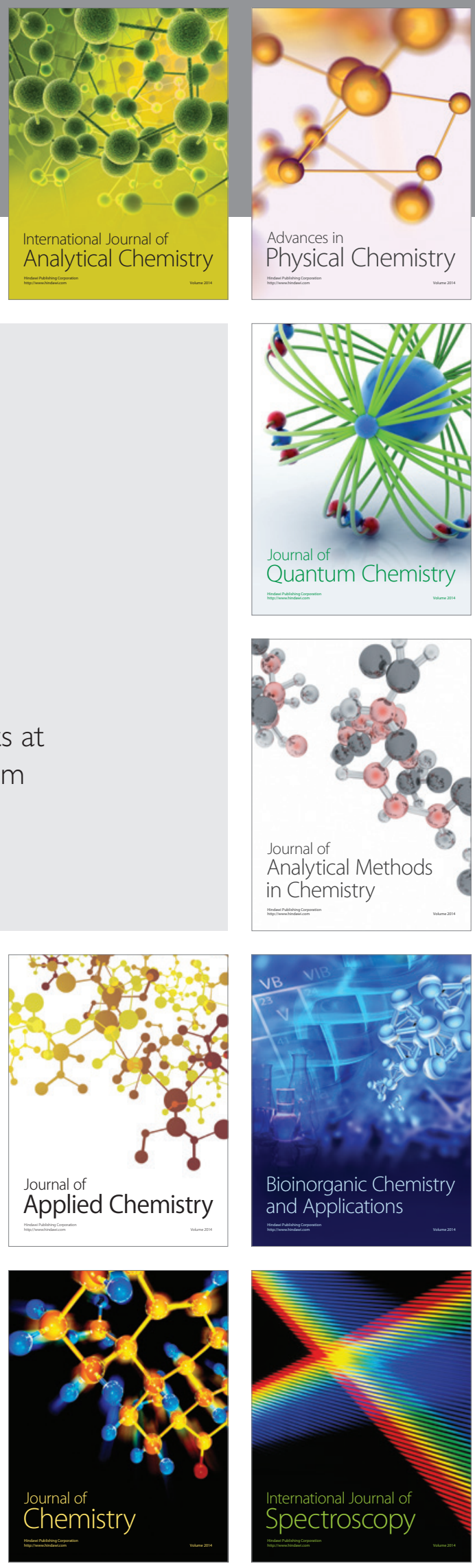